\title{
ENVELHECENDO NA PERCEPÇÃO DAS PESSOAS LONGEVAS ATIVAS E INATIVAS FISICAMENTE
}

\author{
Marize Amorim Lopes ${ }^{1}$ \\ Rodrigo de Rosso Krug² \\ Albertina Bonetti ${ }^{3}$ \\ Giovana Zarpellon Mazo ${ }^{4}$
}

resumo

O estudo analisou a percepção das pessoas longevas, ativas e inativas fisicamente, participantes dos grupos de convivência para idosos de Florianópolis/SC, sobre o processo de envelhecimento humano. Trata-se de uma pesquisa qualitativa realizada utilizando-se a técnica de grupo focal; envolveu 69 mulheres longevas. Aplicou-se

\footnotetext{
1 Graduação, mestrado e doutorado em Educação Física pela Universidade Federal de Santa Catarina na área de Atividade Física e Saúde. Professora Doutora do Departamento de Educação Física, Centro de Desportos/UFSC. E-mail: marize.amorim@ufsc.br

2 Aluno do Curso de Doutorado em Ciências Médicas UFSC. E-mail: rodkrug@bol.com.br

3 Possui mestrado em Educação Física e doutorado em Enfermagem pela Universidade Federal de Santa Catarina, e pós-doutoramento pela Universidade Pablo de Olavide de Sevilha (Espanha). Professora Doutora do Departamento de Educação Física, Centro de Desportos/UFSC. E-mail: a.bonetti@ufsc.br

4 Graduação em Educação Física, mestrado em Ciências do Movimento Humano pela Universidade Federal de Santa Maria e doutorado em Ciências do Desporto pela Universidade do Porto. Professora Doutora do Centro de Ciências da Saúde e Desportos, UDESC. E-mail: giovana.mazo@udesc.br
} 
a entrevista semiestruturada e o Questionário Internacional de Atividade Física, seguidos da análise de conteúdo. Os resultados mostram que a percepção do processo de envelhecimento direciona-se a aspectos relacionados à saúde (limitação por doença, diferentes formas de envelhecimento, problemas cognitivos, esquecimento, medo de quedas e fraturas, idade avançada) e a fatores psicossociais (solidão, preocupações, motivação, experiências positivas, entre outros). Sendo assim, sugere-se que os profissionais da saúde intervenham com ações que promovam a adoção de hábitos de vida mais ativa, visando a manter e melhorar a capacidade funcional da pessoa longeva, em prol do envelhecimento humano mais autônomo e saudável.

palavras-chave

Longevidade. Atividade Motora. Envelhecimento.

\section{Introdução}

Os indivíduos que estão na faixa etária de 80 ou mais anos de idade são denominados de "pessoas longevas". O número total de octogenários no mundo é de aproximadamente 69 milhões, correspondendo a 1\% da população mundial. Conforme o censo de 2010, no Brasil foram identificadas 2.935 .585 (3,23\%) pessoas nesse estrato etário; destas, aproximadamente 80 mil vivem em Santa Catarina, das quais sete mil residem em Florianópolis (IBGE, 2010).

Este cenário populacional instiga o questionamento sobre a percepção da pessoa longeva em seu processo de envelhecimento humano, pois a velhice é cada vez mais presente em nossa sociedade e se processa de diferentes maneiras e ritmos, definindo-se por fatores ambientais, genéticos, biológicos, psicológicos, sociais, culturais, entre outros (ACSM, 2009).

Assim, com base na relevância e atualidade do tema, não seria por demais dizer que a investigação sobre pessoas longevas é um território novo na ciência e, portanto, desafiador (JANNEY et al., 2010). Certamente encontrar-se-ão restrições quanto às fontes de informação ao desenvolvimento da pesquisa qualitativa, uma vez que a maioria dos estudos na área da longevidade humana é de caráter descritivo e quantitativo e, não obstante, envolvem pessoas acima de 55 anos (e não acima de 80 anos, como é o objeto 
em questão). Portanto, os estudos e fontes disponíveis, por não tratarem especificamente da população longeva, podem não refletir a realidade deste grupo etário e, conhecendo esta deficiência, deve-se utilizar com cautela os dados que disponibilizam.

Neste sentido, objetivou-se neste estudo descrever a percepção das pessoas longevas ativas e inativas fisicamente, participantes de grupos de convivência para idosos de Florianópolis/SC, sobre o processo de envelhecimento humano.

\section{Procedimentos metodológicos}

A pesquisa caracterizou-se como qualitativa descritiva exploratória. A mesma ocorreu nos grupos de convivência para idosos cadastrados na Prefeitura Municipal de Florianópolis/SC e os dados foram coletados de setembro de 2010 a abril de 2011. Foram identificadas 493 pessoas longevas matriculadas nestes grupos de convivência para idosos, sendo avaliadas 352 pessoas.

Foram convidadas a participar da pesquisa somente as pessoas longevas do sexo feminino, por serem maioria nos grupos de convivência. Além disso, foram convidadas a participar do estudo 30 mulheres longevas de cada região sanitária de Florianópolis/SC (Continente, Centro, Leste, Sul, Norte), sendo 15 idosas longevas inativas fisicamente com pontuação zero minutos por semana (Grupos Focais Inativos = GFI) e 15 longevas ativas fisicamente com pontuação maior que 300 minutos por semana (Grupos Focais Ativos = GFA), a partir dos resultados do Questionário Internacional de Atividade Física (IPAQ) adaptado para idosos (MAZO; BENEDETTI, 2010).

Assim, participaram do estudo 69 mulheres com 80 anos ou mais de idade, sendo 39 nos GFA (média de idade $=82,51 \pm 2,90$ anos) e 30 nos GFI (média de idade $=86,07 \pm 4,70$ anos). Os GFA e GFI foram distribuídos pelas regiões de Florianópolis, SC e pelos grupos de convivência: GFA1 Centro (n $=09)$, GFA2 Leste $(\mathrm{n}=07)$, GFA3 Sul $(\mathrm{n}=09)$, GFA4 Norte $(\mathrm{n}=07)$ e GFA5 Continente $(n=07)$, GFI6 Centro $(n=6)$, GFI7 Leste $(n=4)$, GFI8 Sul $(n=6)$, GFI9 Norte $(\mathrm{n}=8)$, GFI10 Continente $(\mathrm{n}=6)$.

Para a coleta de informações utilizou-se o Questionário Internacional de Atividade Física forma longa, semana normal, adaptado para idosos (MAZO; BENEDETTI, 2010), que avalia o nível de atividade física (AF); e a técnica de grupo focal, que é uma forma de entrevista com grupos, baseada na comunicação e na interação, por meio de tópico a ser explorado, podendo abordar um tema e um grupo específico, a fim de entender em profundidade 
o comportamento de um determinado grupo (TRAD, 2009). No caso deste estudo a técnica foi importante para entender a percepção das pessoas longevas sobre o processo de envelhecimento humano.

Para analisar o nível de AF das pessoas longevas a partir do IPAQ, foi utilizado o somatório dos tempos (minutos por semana) das AF moderadas e vigorosas nos domínios trabalho, lazer, transporte e tarefas domésticas. Para a o tratamento dos resultados realizou-se a transcrição do áudio da técnica de grupo focal, que fora redigido no programa Microsoft Word e posteriormente introduzido no software ATLAS TI (Qualitative Research Solutions) e interpretado pela análise de conteúdo temática (MINAYO, 2008).

Esta pesquisa foi aprovada pelo Comitê de Ética e Pesquisa em Seres Humanos da Universidade do Estado de Santa Catarina (parecer no 149/2010). Todos os participantes assinaram o Termo de Consentimento Livre Esclarecido.

\section{Resultados e discussão}

A seguir apresentam-se as subcategorias sobre a percepção do processo de envelhecimento que emergiram do estudo; sendo que tanto as pessoas longevas ativas como as inativas assinalaram as mesmas subcategorias (Figura 1).

Figura 1 - Envelhecendo na percepção das longevas nos Grupos Focais.

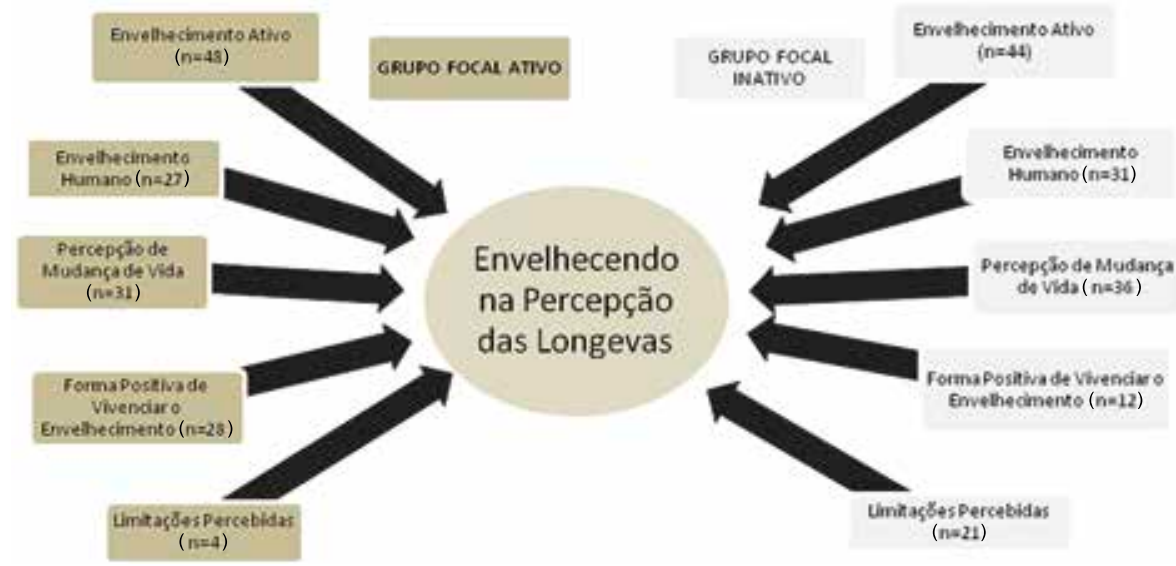

Obs.: o número da amostra foi alterado em função de os participantes terem relatado mais de uma resposta. 
Na percepção das mulheres longevas, o processo de envelhecimento humano sofreu alterações ao longo dos anos. Destacam que esta mudança ocorreu pois as pessoas mais velhas atualmente apresentam menos mudanças físicas oriundas do processo de envelhecimento: "[...] quando me casei a minha sogra tinha 60 anos, já era uma velhinha, sou mais ativa aos 83 anos do que a minha sogra com 60 anos" (GFI9).

O envelhecimento é um processo natural, progressivo e comum a todos, envolvendo mecanismos debilitantes que afetam a capacidade de realizar funções, como as atividades básicas e instrumentais da vida diária, sendo que ele varia de pessoa para pessoa e é determinado pela genética, influências do estilo de vida, meio ambiente e estado nutricional (ACSM, 2009).

Ao longo da vida é possível identificar mudanças biopsicossociais entre as fases de desenvolvimento humano (HIRVENSALO; LINTUNEM, 2011). As longevas observam o envelhecimento como um processo contínuo: “[...] Fico realizada, porque eu não parei. Envelhecer não é parar, porque eu não parei esperando o tempo passar. Eu fui muito além" (GFA5).

As idosas percebem que o envelhecimento exige mudança e cautela em suas ações: "[...] tudo tem que ser feito na medida do possível, não se deve exigir e querer fazer aquilo que realizava quando jovem, tem que ter calma, faço sempre tudo 'devagarzinho'" (GFA4). À medida que aumenta a idade cronológica, as pessoas se tornam menos ativas e suas capacidades físicas diminuem, surgindo as doenças crônicas (HIRVENSALO; LINTUNEM, 2011). Um grande aliado da longevidade é a manutenção de um estilo de vida ativo (PATRÍCIO et al., 2008).

\subsection{Limitações percebidas}

As limitações são sentidas e observadas com o surgimento das doenças crônicas. As longevas buscam se adaptar com essa nova realidade. "[...] tenho artrose e dor no pé. É, mas mesmo com dor eu faço minhas atividades" (GFA5); “[...] diabetes, pressão alta, colesterol alto. Isso é tudo doença de velho" (GFI8).

As doenças comumente associadas a esta faixa etária mais velha são o acidente vascular encefálico, câncer, doenças cardiovasculares, hipercolesteromia e hipertensão (IBGE, 2010). Os déficits auditivos e discreta perda de memória têm grande prevalência nas pessoas longevas (SCHMIDT et al., 2009). 
As alterações funcionais próprias do envelhecimento associadas às doenças crônicas contribuem também para as incapacidades (HIRVENSALO; LINTUNEN, 2011).

Envelhecer com saúde é a meta de todas as pessoas longevas. A percepção sobre a velhice e a transformação que se processa ao longo dos anos é sentida em alguns momentos da vida: “[...] o meu envelhecimento está se agravando. Fui à minha médica e ela mandou tomar mais dois remédios, eu tomava quatro para o coração, quatro para diabetes, colesterol e reumatismo" (GFI10). Contudo, em outros momentos esquecem a idade que tem: "[...] considero-me velha quando tenho algum problema de saúde, mas normalmente não me considero velha [...]" (GFI10). A idade subjetiva que a pessoa sente ter, seja do ponto de vista mental, físico ou social, pode ser influenciada com o avançar da idade pelos papéis e situações a serem enfrentados.

As pesquisadas percebem seu envelhecimento e apontam algumas causas que incomodam: o esquecimento, a solidão, a preocupação com familiares, as doenças, entre outras. O sentir-se só é um sentimento comum entre as pessoas longevas. Alegam: "[...] a pessoa na solidão envelhece mais rápido" (GFI8). Mas, por outro lado, a aceitação do seu processo de envelhecimento contribuirá para uma vida melhor. "[...] a pessoa tem que aceitar seu envelhecimento, sinto orgulho de ter 83 anos e quero chegar mais com saúde, pois tenho energia" (GFI10). No processo de envelhecer, as pessoas idosas deparam-se com novos desafios e atitudes relacionados às mudanças físicas e sociais; sendo assim, pode-se observar que a aquisição de hábitos mais saudáveis ajuda a atingir a longevidade com mais qualidade (ACSM, 2009).

$\mathrm{Na}$ visão das longevas, o envelhecimento se processa diferenciadamente e destacam a forma de ser e de interagir no meio como um fator predominante para as diferentes formas de se envelhecer encontradas em nossa sociedade. Relatam que tudo é relativo: “[...] depende do organismo da pessoa, tenho uma irmã que é a mais nova de todas, está internada com um monte de doença, uma dura mais e a outra menos, uma tem doença e a outra não" (GFI8). O envelhecimento humano, portanto, ocorre de forma diferenciada entre os indivíduos, podendo ter influência genética, psicológica, social, cultural, entre outros (ACSM, 2009).

Outro aspecto evidenciado nas falas das longevas foi quanto às oportunidades vivenciadas. A pessoa ser calma e tranquila foram fatores destacados para a longevidade humana: "[...] minha avó viveu 100 anos porque ela era bem calma, pessoa do bem, instruída, tudo para ela estava bom, era difícil se incomodar" (GFI8). 
O envelhecimento do homem merece destaque nesta análise, uma vez que as pesquisadas salientam que os homens morrem mais cedo, pois não se cuidam, são mais sedentários: "[...] os homens não se cuidam igual à mulher, fumam mais e as mulheres vão mais ao médico. O meu marido não gostava de sair, não dançava" (GFA2). Esses são alguns dos motivos que justificam o número significativo de mulheres nos grupos de convivência. A longevidade se faz mais presente nas mulheres do que nos homens. Estudos sobre a mortalidade no Brasil no período de 1991 a 2007 revelaram que o diferencial por sexo, em 1991, era de 7,70 anos, sendo que houve uma discreta diminuição em 2007, passando para 7,62 anos (IBGE, 2008). Embora as mulheres sejam mais suscetíveis a contrair doenças incapacitantes, essas estão mais preparadas para enfrentar as doenças do que os homens (IBGE, 2010).

\subsection{Forma positiva de vivenciar o envelhecimento}

A forma de vivenciar as transformações e as doenças adquiridas por causa do processo envelhecimento foi evidenciada pelas longevas destacando estratégias de ação que estabelecem para uma melhor adaptação de vida: “[...] tenho consciência que as atividades que me alegraram por muito tempo, agora não dá mais para fazer por causa de minhas limitações, mesmo assim prossigo pela vida" (GFA1); "[...] a norma é: se doer tem que mexer mais ainda, eu não tenho dor porque me mexo bastante" (GFA5); “[...] sou feliz graças a Deus, tenho um pouquinho de saúde, porque ainda ando, estou com dificuldade de caminhar sozinha" (GFI10).

Estudo de Patrício et al. (2008) demonstrou que mesmo sentindo as transformações que a vida impõe com a longevidade, o ser humano luta constantemente para viver mais e melhor. A adoção de um estilo de vida ativo pela pessoa longeva é parte fundamental para que esta envelheça com saúde. A prática de atividade física está associada também com uma melhor mobilidade, capacidade funcional e qualidade de vida durante o envelhecimento.

As diferentes maneiras de se adaptar e transpor esses obstáculos, bem como as experiências de vida, vêm contribuindo para identificar as distintas formas de envelhecimento. As longevas assinalam que: "[...] a pessoa tem que viver, tudo é consequência do que viveu" (GFA1); "[...] a vida está boa e que não está melhor porque sempre aparece algum problema" (GFI10). Percebe-se, apesar das perdas funcionais, orgânicas e mentais, que a pessoa idosa que tenha tido hábitos de vida saudáveis pode vir a ser uma pessoa com 
autossuficiência para tarefas diárias e com capacidade adaptada para manter relações intelectuais e sociais com o meio que a rodeia.

Praticar atividade física regular, ter uma alimentação adequada, controlar o estresse, manter relacionamentos positivos e investir em comportamentos preventivos são hábitos de vida fundamentais para se ter qualidade de vida (HIRVENSALO; LINTUNEN, 2011). As longevas salientam: “[...] a gente está vivendo mais por causa da alimentação, do modo de vida, participação nos grupos" (GFI7). Relataram, ainda, hábitos saudáveis de vida, com realização de AF e nutrição com dieta hipocalórica mediterrânea rica em azeite de oliva, fatos que podem também ter contribuído para a sua longevidade (CHAIM; IZZO; SERA, 2009).

A aceitação de seu próprio envelhecimento, bem como administrar os seus sentimentos, vem favorecendo as pessoas longevas a viver essa etapa de vida. "[...] olhar no espelho e se gostar" (GFA4); “[...] não quero guardar tristeza, nem sofrimento, quem já sofreu muito quando nova agora a vida está boa, agora o que me resta é só alegria, amizade e passear" (GFA1). O entendimento das transformações oriundas do processo de envelhecimento é um fator essencial para um envelhecimento bem-sucedido; assim como a prevenção de doenças, as mudanças de hábitos e estilos de vida prejudiciais à saúde, o estabelecimento de relações sociais e familiares e o cultivo do bom humor, contribui significativamente para o aumento da qualidade de vida (ACSM, 2009).

\subsection{Percepção de mudança de vida}

As longevas pesquisadas compreendem que houve muitas mudanças na vida das pessoas, comparando-se aos tempos atuais. Relatam que antigamente as mulheres não saíam de casa, só cuidavam da casa, dos filhos e maridos e destacam que viviam para trabalhar. As atividades eram pesadas e cansativas. "[...] Não tinha máquina, as roupas eram lavadas sempre à mão, forçando o braço" (GFA5). Elas ressaltaram também o surgimento da água encanada, auxiliando no banho e limpeza da casa. Quanto à energia elétrica ressaltam: "[...] acendíamos a lamparina conhecida como pomboca e amanhecíamos com o nariz todo preto da fumaça inalada [...]" (GFI6).

Os meios de comunicação eram bem precários, não existiam televisão nem rádio, em consequência alegam que o tempo custava a passar. O relacionamento familiar e de vizinhos era diferente. Hoje falta respeito, confiança e amor entre as pessoas: "[...] não se tem mais confiança nas pessoas como 
antigamente" (GFA3); "[...] não existe mais respeito, não respeitam mais o idoso" (GFI6); "[...] existia mais amor entre família, foi ficando tudo longe um do outro. Tem pessoa que a gente tem saudade, fica muito tempo sem se ver" (GFI9).

No ponto de vista das longevas o casamento também era diferente: “[...] casou, acabou, era muito sacrifício, a vida hoje é boa" (GFI9). Comentam também que fugiam para depois casar. Quando ficavam viúvas dificilmente casavam novamente, alegando que casamento era somente o primeiro.

Outra percepção comparativa é que hoje tem mais atividades e oportunidade de diversão para as pessoas longevas. "[...] nossas mães não tiveram o privilégio de ter as atividades para idosos que temos hoje" (GFA5); “[...] hoje a pessoa de idade se diverte mais que antes [...] antigamente não tinha ginástica, caminhada e grupo de idosos" (GFI8).

Todas essas mudanças de vida apontadas e contextualizadas nos grupos focais fazem refletir sobre como estas possibilidades apresentadas nos dias atuais promovem uma vida com mais qualidade: "[...] usufruir de todas as regalias que temos hoje em dia, você tem um grupo, viaja, conhece lugares e pessoas" (GFI7). Assim sendo, declararam serem mais felizes nos dias de hoje.

\subsection{Envelhecimento ativo}

A participação efetiva de pessoas longevas em atividades de lazer caracteriza um hábito de vida ativo que deve ser estimulado (VON BONSDORFF; RANTANEN, 2011). Envelhecer de forma ativa e ocupar-se com atividades prazerosas favorecem as pessoas longevas a uma vida com mais qualidade. A pessoa deve se envolver espontaneamente com atividades ocupacionais que favoreçam a sua participação social voluntária, bem como a sua capacidade criativa, sejam essas para repousar, divertir-se e recrear-se (ACSM, 2009). Manter-se em plena atividade diferencia as pessoas longevas: "[...] a pessoa, ao entrar no grupo, conversa, conhece outras pessoas, adapta-se a sua realidade e idade" (GFA1).

Existe uma forte associação do envelhecimento ativo, nas pessoas longevas, com indicadores de autonomia e mobilidade física. As longevas ativas fisicamente declaram que são muito ativas e que não param nunca. Entre as atividades que fazem destacaram: pular corda, fazer ginástica, caminhada, natação, hidroginástica, viajar, passear, participar de grupos de convívio, fazer cálculo de cabeça. Estar em plena atividade as deixa com mais disposição para continuarem fazendo e vivendo intensamente: “[...] não 
parar, movimentar-se, fazer alguma atividade, fazer ginástica, é viver ativamente" (GFA4); "[...] não ficar parada, só dentro de casa, é procurar oportunidades nos grupos, o que puder fazer para não ficar atrofiada" (GFA3).

Como as evidências epidemiológicas sustentam o efeito positivo de um estilo de vida ativo para saúde da pessoa idosa, a atividade física vem sendo considerada como um integrante fundamental dos programas de promoção de saúde na velhice (OMS, 2002). A pessoa idosa busca atividade física para uma vida mais saudável, procura atividades como a ginástica, dança e caminhada (LIMA; SILVA; GALHARDONI, 2008).

As longevas inativas fisicamente também apontam que não se deve ficar parada. Alegam que: "[...] tem que trabalhar em casa, fazer alguma coisa, ficar parado dormindo não dá" (GFI6); “[...] a gente não pode parar, tem que se mexer, pois senão a gente fica enferrujada" (GFI7). Elas relataram gostar de participar de grupos, palestras, de passear, viajar, sair de casa, andar, pintar, bordar, fazer crochê e tricô e jogos de canastra. Manter-se ativo, mais do que nunca, passou a ser um dos fatores mais determinantes para a promoção de saúde dos idosos (HIRVENSALO; LINTUNEN, 2011).

Percebe-se que o centro de interesse em relação às atividades realizadas que perfaz o envelhecimento ativo das longevas se diferencia, pois as longevas ativas destacaram realizar atividades com intensidade moderada, já as inativas relataram atividades de baixa intensidade. As atividades que realizavam em comum foram passeios, viagens e participação nos grupos de convivência.

A autonomia de realizar atividades sozinhas, tomar decisões e administrar sua vida independentemente foi enaltecida pelas longevas ativas. Ficam satisfeitas por ter essa possibilidade. "[...] faço muitas coisas sozinha, cozinho, passo a minha roupa, não dependo de ninguém” (GFA1); “[...] moro sozinha, tenho um monte de filho, mas faço tudo sozinha, resolvo todos os meus problemas" (GFA4).

Elas gostam de realizar atividades de lazer: "[...] o que nós fazemos é sair, dançar, fazer ginástica, viajar, encontrar as amigas, ir ao cinema, teatro" (GFA1). O importante é que a pessoa idosa esteja em grupo e dê continuidade, fazendo com que supere as dificuldades biopsicossociais decorrentes do processo de envelhecimento (WITTER, 2006).

As longevas relataram gostar de cuidar do visual e da saúde: "[...] não sei sair sem brinco, sem colar, não sei sair sem nada" (GFA4); "[...] sempre me cuidei muito, o melhor remédio é fazer ginástica e caminhar" (GFA4). O autocuidado evidenciado pelas longevas ativas confirma o papel do exercício físico na relação com o aumento da alegria, da autoeficácia e do autoconceito. Observa-se, assim, que atividades físicas podem promover à pessoa uma 
sensação de sucesso, e, desta forma, reforçam a autoimagem e uma autoestima positiva (MAZO; CARDOSO; AGUIAR, 2006).

As longevas têm como hábito caminhar regularmente, utilizando a caminhada como meio de locomoção: "[...] gosto de ir ao grupo a pé" (GFA4); "[...] vou de minha casa até o grupo a pé, vou e volto [...]" (GFA2). A caminhada é um esforço físico seguro, pois apresenta reduzidos riscos de lesões cardiovasculares e ortopédicas, é uma atividade de simples execução e de fácil adaptação, permitindo, assim, uma melhor aceitação por parte das longevas (KRUG et al., 2011).

Promover o envelhecimento ativo é um fato constatado nos depoimentos durante os grupos focais, manter-se ocupadas durante toda a semana com atividades que lhes fazem bem: "[...] gosto de atividade, quanto tem ginástica vou fazer, depois volto, faço almoço e à tarde faço jantar para filha e a espero chegar do serviço" (GFA3). A meta das pessoas longevas é promover uma vida saudável e com qualidade; para tanto, cultivam atitudes para se relacionar bem: "[...] tem que ter paciência. Devendo saber escutar e respeitar" (GFI9). Enfatizam, também, que devem manter-se ativas: "[...] acho que nós necessitamos tanto do exercício físico como precisamos da alimentação para sobreviver, ambos têm a mesma importância" (GFA2).

\section{Considerações finais}

Com os resultados obtidos, pode-se inferir que a percepção do processo de envelhecimento das pessoas longevas direciona-se aos aspectos relacionados à saúde, a limitação pela doença, o envelhecer de forma diferenciada e positiva, além de ser ativo fisicamente.

Os resultados obtidos mostram em vários momentos das falas das pessoas longevas a arte de viver em uma idade longeva, consistindo na aplicação de estratégias de ação para compensar as limitações oriundas da idade avançada, das doenças, do meio ambiente e social, e também estratégias de promoção de saúde. Sendo assim, deve-se possibilitar à pessoa longeva um suporte que a ajudará a manejar com eficácia sua vida, bem como permitir à mesma ter saúde para gerenciar e decidir seu próprio destino. Qualquer pessoa em idade avançada deve ser capaz de conduzir sua própria vida, ter autonomia e determinar quando, como e onde se darão suas atividades de trabalho, convívio social e lazer.

A sociedade deve se preocupar em oferecer às pessoas longevas ações de políticas sociais, psicológicas e de saúde preventiva e curativa e estímulos 
corporais por meio de práticas diárias de atividade físicas adequadas para que possam ter novas formas de seleção, otimização e compensação das oportunidades para a promoção de saúde, participação e segurança para o bem estar biopsicossocial ao longo do processo de viver da pessoa longeva.

\author{
AGING IN THE PERCEPTION OF ELDERLY \\ PEOPLE PHYSICALLY ACTIVE AND INACTIVE
}

\title{
abstract
}

The study examined the perception of physically active and inactive longevous people, participants from elderly social groups in Florianópolis/SC, about the human aging process. A qualitative research was developed using the focus group technique; it involved 69 elderly women. A semi-structured interview and the International Physical Activity Questionnaire were applied, followed by a content analysis. The results show that the perception of the aging process points to health-related aspects (limitation by disease, different forms of aging, cognitive problems, forgetfulness, fear of falls and fractures, advanced age) and psychosocial factors (loneliness, concerns, motivation, positive experiences, among others). Therefore, it is suggested that health professionals take actions to promote a more active lifestyle, in order to maintain and improve the functional capacity of the longevous person, in favor of a healthier and more autonomous human aging.

keywords

Longevity. Physical Activity. Aging.

\author{
referências
}

\begin{abstract}
AMERICAN COLLEGE OF SPORTS MEDICINE (ACSM). Exercise and Physical Activity for Older Adults. Medicine Science of Sports and Exercise, Indianapolis, v. 41, n. 7, p. 1510-1530, July 2009.

CHAIM, Janice; IZZO, Helena; SERA, Celisa Tiemi Nakagawa. Cuidar em saúde: satisfação com imagem corporal e autoestima de idosos. O Mundo da Saúde, São Paulo, v. 33, n. 2, p. 175-181, fev. 2009.

HIRVENSALO, Mirja; LINTUNEN, Taru. Life-course perspective for physical activity and sports participation. European Review of Aging and Physical Activity, Munich, v. 8, n. 1, p. 13-22, Jan. 2011
\end{abstract}


INSTITUTO BRASILEIRO DE GEOGRAFIA E ESTATÍSTICA (IBGE). Síntese de indicadores sociais - Uma análise das condições de vida da população brasileira. 2010. Disponível em: <http://www.ibge.gov.br/home/presidencia/noticias/imprensa/ppts/0000000144. pdf>. Acesso em: 26 jun. 2011.

Projeção da População do Brasil por Sexo e Idade: 1980-2050. 2008. Disponível em: <http://uww.ibge.gov.br/home/estatistica/populacao/projecao_da_populacao/2008/projecao.pdf>. Acesso em: 12 jun. 2010.

JANNEY, Carol et al. Longitudinal Physical Activity Changes in Older Men in the Osteoporotic Fractures in Men Study. Journal American Geriatrics Society, Malden, v. 58, n 6, p. 1128-1133, June 2010.

$K R \cup G$, Rodrigo de Rosso et al. As contribuições da caminhada como atividade física de lazer para idosos. Licere, Belo Horizonte, v. 14, n. 4, p. 1-29, dez. 2011.

LIMA, Ângela Maria Machado; SILVA, Henrique Salmazo da; GALHARDONI, Ricardo. Envelhecimento bem-sucedido: trajetórias de um constructo e novas fronteiras. Comunicação, Saúde e Educação, Botucatu, v. 12, n. 27, p. 795-807, out./dez. 2008.

MAZO, Giovana Zarpellon; BENEDEITI, Tania Rosane Bertoldo. Adaptação do questionário internacional de atividade física para idosos. Revista Brasileira de Cineantropometria e Desempenho Humano, Florianópolis, v. 12, n. 6, p. 480-484, jun. 2010.

MAZO, Giovana Zarpellon; CARDOSO, Fernando Luiz; AGUIAR, Daniela Lima. Programa de hidroginástica para idosos: motivação, auto-estima e auto-imagem. Revista Brasileira de Cineantropometria e Desempenho Humano, Florianópolis, v. 8, n. 2, p. 67-72, fev. 2006.

MINAYO, Maria Cecília de Souza. O desafio do conhecimento: pesquisa qualitativa em saúde. 11. ed. São Paulo: Hucitec, 2008.

ORGANIZACIÓN MUNDIAL DE LA SALUD (OMS). Envejecimento activo: un marco político. Revista Espanhola de Geriatria e Gerontologia, Barcelona, v. 37, n. S2, p. 74-105, fev. 2002.

PATRÍClO, Karina Pavão et al. O segredo da longevidade segundo as percepções dos próprios longevos. Revista Ciência e Saúde Coletiva, São Paulo, v. 13, n. 4, p. 11891198, jul./abr. 2008.

SCHMIDT, Jaqueline Angela et al. Aplicação do teste do relógio em octogenários e nonagenários participantes de estudo realizado em Siderópolis/SC. Revista Psico, Porto Alegre, v. 40, n. 4, p. 525-530, out./dez. 2009.

TRAD, Leny Bomfim. Grupos focais: conceitos, procedimentos e reflexões baseadas em experiências com o uso da técnica em pesquisas de saúde. Physis, Rio de Janeiro, v. 19, n. 3, p. 777-796, mar. 2009.

VON BONSDORFF, Mikaela; RANTANEN, Taina. Progression of functional limitations in relation to physical activity: a life course approach. European Review of Aging and Physical Activity, Munich, v. 8, n. 1, p. 23-30, Apr. 2011.

WITTER, Geraldina Porto. Envelhecimento: referências teóricas e pesquisa. 1. ed. Campinas: Alínea, 2006. 